\title{
Towards Large Area CVD Diamond Disks for Brewster-Angle Windows
}

\author{
G. Aiello ${ }^{\mathrm{a}}$, S. Schreck ${ }^{\mathrm{a}}$, K.A. Avramidis ${ }^{\mathrm{b}}$, T. Franke, ${ }^{\mathrm{c},}$, G. Gantenbein ${ }^{\mathrm{b}}$, J. Jelonnek ${ }^{\mathrm{b}}$, A. Meier ${ }^{\mathrm{a}}$,

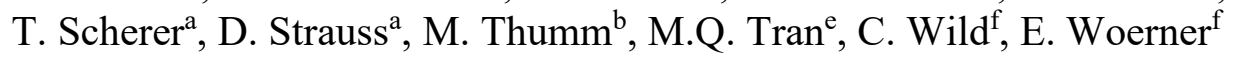 \\ ${ }^{a} I A M,{ }^{b} I H M$, KIT, Hermann-von-Helmholtz-Platz 1, 76344 Eggenstein-Leopoldshafen, Germany \\ ${ }^{c}$ EUROfusion Consortium, Boltzmannstrasse 2, 85748 Garching, Germany \\ ${ }^{d}$ Max-Planck-Institut für Plasmaphysik, Boltzmannstrasse 2, 85748 Garching, Germany \\ ${ }^{e}$ Swiss Plasma Center (SPC), EPFL, 1015 Lausanne, Switzerland \\ fDiamond Materials GmbH, Hans-Bunte-Strasse 19, 79108 Freiburg, Germany
}

\begin{abstract}
In the frame of the EUROfusion Work Package Heating and Current Drive (WP HCD) of the Power Plant Physics and Technology (PPPT) program, CVD diamond disk Brewster-angle windows for gyrotron operation at multi-megawatt RF power levels and long pulses are under development. These windows allow for frequency steptuneable operation. The Brewster-angle of $67.2^{\circ}$ for diamond leads to an elliptical connection of the disk to the copper waveguides (WGs), requiring an advanced joining process. For proper transmission of the RF power, the disk consists of low loss CVD diamond of optical grade. The current target for the WG aperture of DEMO is $63.5 \mathrm{~mm}$. It allows for an RF power transmission of $2 \mathrm{MW}$, but it requires a disk diameter of $180 \mathrm{~mm}$ for the $67.2^{\circ}$ angle. In addition, a thickness of approximately $2 \mathrm{~mm}$ is needed to achieve the proper mechanical stability. State of the art microwave plasma reactors are not capable of growing disks of such size. The maximum available diameter of a polycrystalline CVD diamond disk suited to microwave applications is currently $140 \mathrm{~mm}$. Thus, the industrial partner Diamond Materials GmbH (Freiburg, Germany) is doing extensive diamond growth experiments. A first of its kind, $180 \mathrm{~mm}$ thermal grade, crack-free, diamond disk was produced in the microwave plasma reactor with an average unpolished thickness of about $2 \mathrm{~mm}$. First loss tangent measurements have been also performed. This paper describes the steps and the first results of this non-straightforward path, a challenging new field for diamond manufacturers and a major breakthrough for future frequency step-tuneable operation.
\end{abstract}

Keywords: DEMO, EC system, Brewster diamond window, Diamond growth, Large aperture

\section{Introduction}

In the ITER Electron Cyclotron Heating and Current Drive (EC HCD) system, the gyrotrons, which are the microwave sources, generate beams at fixed frequency of $170 \mathrm{GHz}$ with a radio frequency (RF) output power of $1 \mathrm{MW}$ [1]. A diamond window, a component formed by a Chemical Vapour Deposition (CVD) diamond disk integrated in a metallic housing, acts as vacuum barrier at the output of the gyrotron (gyrotron window) and also it is used close to the tokamak as vacuum, tritium and, in case of failure, overpressure boundary (tokamak window). Beyond ITER, in the path towards the European concept of a demonstration power plant (EU DEMO) [2], the current target is to have gyrotrons which are able to allow 2 MW dual-frequency operation $(170 / 204 \mathrm{GHz})$ and/or frequency step-tunability in 2$3 \mathrm{GHz}$ over a range of $\pm 10 \mathrm{GHz}$, even above $200 \mathrm{GHz}$ (e.g., $\sim 240 \mathrm{GHz}$ ) [3]. It should be noted that gyrotrons cannot be tuned continuously.

Broadband diamond window solutions that are able to withstand the transmission of high power microwave beams are thus required. Since 2014, a strong research \& development (R\&D) activity, coordinated by the PPPT department of the EUROfusion Consortium, has been carried out on the HCD gyrotrons to fulfil the requirements imposed by DEMO on such microwave sources. In the ITER gyrotrons, the diamond disk of the window is perpendicular to the beam direction and it has a diameter of $106 \mathrm{~mm}$ and a resonant thickness of $1.85 \mathrm{~mm}$, corresponding to 5 times $\lambda / 2$ (the half wavelength) of the microwave beam inside the diamond material. After passing the tokamak window, the EC beam needs to be steered by moveable mirrors, facing the ITER plasma, to deposit the EC power at defined magnetic flux surfaces in the plasma. On the contrary, with broadband windows, the RF beam is launched at a fixed angle without any movable parts close to the plasma and a different radial power deposition is achieved by fast tuning (in the range of few seconds) the frequency of the gyrotrons [4].

With particular reference to the frequency steptunability feature, in the context of the gyrotron $R \& D$, the window solution under investigation for long pulse operation is the Brewster-angle diamond RF window, which has a wide transmission band [5]. For $2 \mathrm{MW}$ power transmission, such a window requires an optical grade CVD diamond disk of a minimum diameter of $180 \mathrm{~mm}$ and thickness of about $2 \mathrm{~mm}$, while the maximum available diameter of a diamond disk in the market suited to microwave applications is currently $140 \mathrm{~mm}$. It poses thus the primary fundamental challenge in this window development path, to solve with the essential support of the industrial partner Diamond Materials GmbH in Freiburg, Germany [6], 
where extensive diamond growth experiments are ongoing.

This is a new field for diamond manufacturers that requires dedicated technological solutions and obviously an intense research activity. In this paper, the steps already done and ongoing in the large area diamond disks development are described and discussed together with the challenges, the first important achievements and the first loss tangent $(\tan \delta)$ measurements, aiming to check the diamond quality from the microwave transmission perspective.

\section{Brewster-angle window concept}

As shown in Fig. 1a, the Brewster window concept consists of a polycrystalline diamond disk joined to copper WGs at the Brewster angle of $67.2^{\circ}$ for diamond. It can be immediately observed that for a small WG aperture of only $50 \mathrm{~mm}$, a diamond disk of $140 \mathrm{~mm}$, corresponding to the current technological limit for optical grade CVD diamond growth, is already required. A Brewster window with $50 \mathrm{~mm}$ aperture was already successfully implemented and tested in a MW-class, Dband, step-frequency tuneable gyrotron prototype, but only for short pulses (a few ms) and therefore without any cooling layout [7].

In the present case, where the target for the WG aperture is $63.5 \mathrm{~mm}$ to allow the transmission of $2 \mathrm{MW}$ $\mathrm{RF}$ power, a minimum diameter of $180 \mathrm{~mm}$ is thus required for the diamond disk due to its inclined position. Besides, a thickness of about $2 \mathrm{~mm}$ is needed to assure the proper structural stability, considering also accidental events like overpressure of 2 bar across the disk.

a)

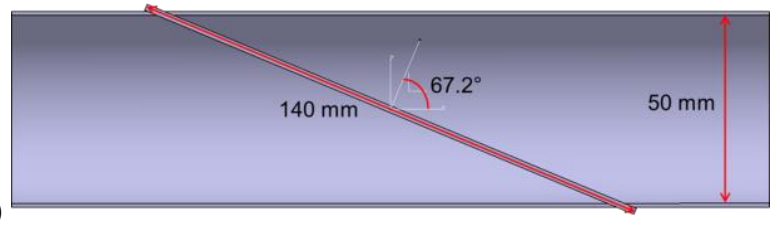

b)

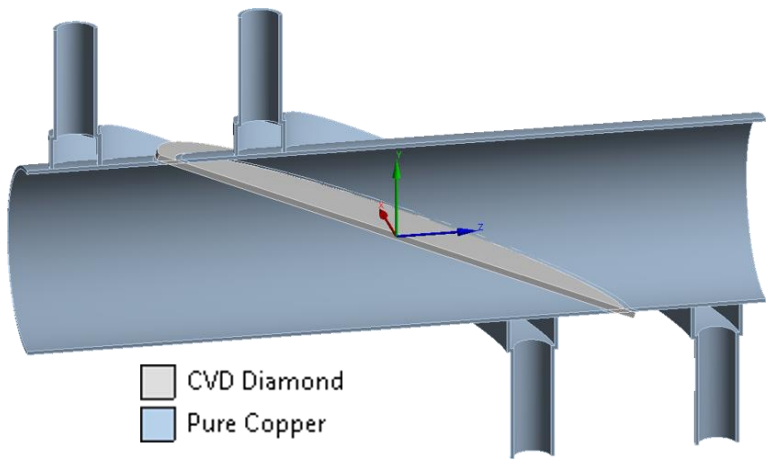

Fig. 1. Diamond Brewster window (a) and a conceptual cooling layout (b) to remove the heat absorbed in the disk.
In theory, given a certain thickness of the disk at the Brewster configuration, assuming a beam linearly polarized in the plane of incidence (parallel polarization), the RF power is transmitted for all possible frequencies without any reflection. In reality, there is always a small reflection depending on the RF beam mode and therefore, the minimum reflection condition shall be also taken into account in the thickness calculation [5]. In fact, the output beam of a gyrotron is never perfectly linearly polarized and the resulting orthogonal component of the polarization, in the Brewster configuration, is partially reflected with potential increase of the inner stray radiation. It is worth to mention that, according to calculations based on [8], a thickness of $2.008 \mathrm{~mm}$ of the diamond disk would guarantee the minimum reflection of such orthogonal polarization at the frequencies of interest in this work, i.e. $170 / 204 / 238 \mathrm{GHz}$.

Within the EUROfusion PPPT Work Package (WP) $\mathrm{HCD}$, the first goal is therefore to show the feasibility of manufacturing these large area optical grade diamond disks. The disks, produced in circular shape, would be then laser cut to an elliptical shape before being brazed to the WGs. In fact, there is no need for a large size of the disk in the transverse direction of the beam. The joining disk-WGs and an efficient cooling of the window represent the two following challenges to solve by dedicated prototyping activities.

The brazing process is carried out at about $900{ }^{\circ} \mathrm{C}$ in a vacuum oven and then the temperature of the involved parts is decreased down to room temperature. Copper and diamond have very different thermal expansion coefficients (ratio of 16 to 1) and this leads to the generation of high stresses in the window. This joining, made already peculiar by the window asymmetry, becomes even more complicated for very large disks. Different types of supports shall be experimentally investigated to keep the parts aligned and reduce the resulting stresses at the minimum.

Former FEM thermal and structural analysis results have given some indications on the cooling layout of the window in order to remove the heat absorbed in the disk [9]. However, the final choice shall be based on a manufacturing feasibility study. An example of a cooling layout is shown in Fig. 1b with external elliptical channels. It is clear from the analyses that the channels have to follow the position of the disk, otherwise the temperature at the disk center achieves a temperature in the order of $400{ }^{\circ} \mathrm{C}$, well above the conservative limit of $250{ }^{\circ} \mathrm{C}$ for diamond. Different beam power and frequency scenarios were investigated. Fig. 2 shows the temperature profiles along the major axis of the elliptical disk $(140 \mathrm{~mm})$ for these beam scenarios with reference to the geometry in Fig. 1b. It can be noted that, even in the worst case scenario $(2 \mathrm{MW} / 240 \mathrm{GHz})$, the maximum temperature can be safely accepted. For the $180 \mathrm{~mm}$ disks, such analyses are not yet performed, but temperatures in the disk lower than $250^{\circ} \mathrm{C}$ are also expected. 


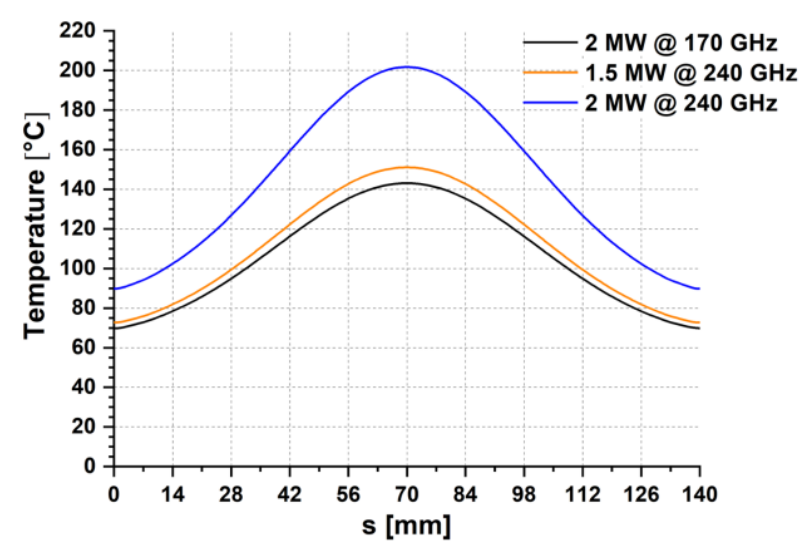

Fig. 2. Temperature profiles along the major axis of the diamond disk for different beam scenarios when external elliptical cooling channels are used [9].

\section{Diamond joining by hot filament method}

In close collaboration with Diamond Materials $\mathrm{GmbH}$, the first attempt to meet the need of large area diamond disks was to adopt, in a small scale, an unconventional method [4]. The basic idea was to join diamond fragments by overgrowing the joint gap with CVD diamond in order to obtain a mechanically stable joint with low absorption.

In previous diamond joining pre-tests, it was observed that the amount of reactive species (i.e. the species leading to the nucleation of diamond crystallites in the substrate) in the joint gap played a major role. For this reason, with respect to other methods, the hot filament method [10] was used for the joining experiments. In fact, as shown in Fig. 3a, the filament can be placed right in the center of the v-groove geometry, chosen to join two diamond plates, to guarantee enough reactive species at the very bottom of the gap.

After several runs for parameters optimization in a dedicated reactor, designed and assembled on purpose by Diamond Materials, the overgrowth of two plates turned to be successful as after 150 hours of diamond deposition (Fig. 3b), the plates grew together and formed a solid compound (better shown in Fig. 3c). However, this attempt was not judged as the path to take towards large Brewster-angle diamond disks. In fact, after 150 hours, only $20 \%$ of the gap was filled up. The growth rate is very low $(0.1$ to $1 \mu \mathrm{m} / \mathrm{h})$ and, moreover, high microwave absorption in the joint gap are expected as atoms of the filament enter anyway the diamond lattice.

\section{Large area disks in microwave plasma reactor}

The target of producing large area diamond disks for microwave applications can be met only by the attempts of growing such disks directly in microwave plasma reactors, where the growth rate is in the range 0.1 to $10 \mu \mathrm{m} / \mathrm{h}[10]$. a)

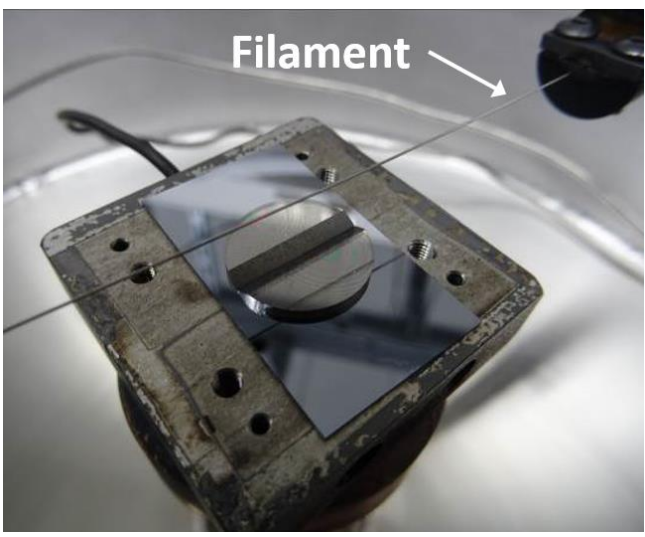

b)

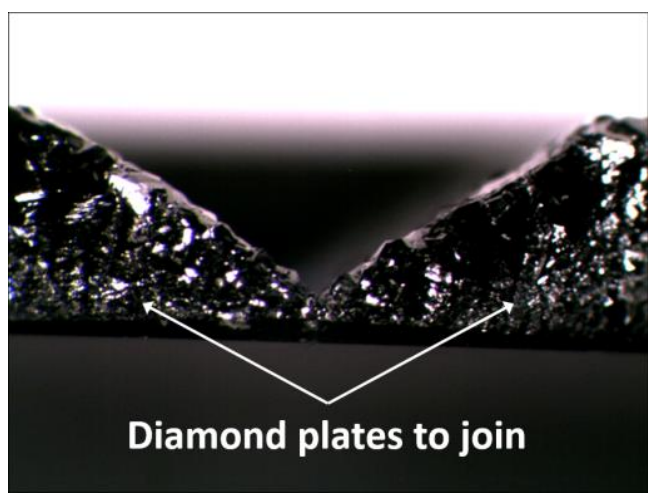

Diamond deposition

c)

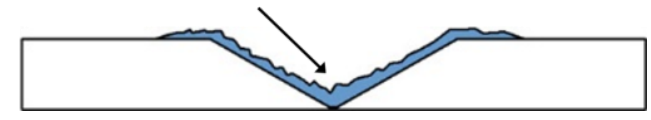

Fig. 3. Diamond plates ready to be joined by the hot filament method (a) and resulting compound (b), better described by the model in (c). The position of the filament in (a) is not adjusted in height.

As already mentioned, a diameter of $140 \mathrm{~mm}$ is for the moment the limit for optical grade CVD diamond growth. In these reactors, aiming to enlarge disk thickness and diameters, the main challenges are that the quality $(\tan \delta)$ from the microwave transmission perspective reduces while it increases the probability to generate cracks that might lead to failure of the grown disks.

In addition, there is the challenge to obtain for such a large disk area a certain homogeneity in the developing thickness distribution. This ensures an acceptable polishing operation at the growth side of the disk, to obtain the wished thickness of the disk after polishing. In the frame of the EUROfusion WP HCD, these challenges have been addressed by several ongoing diamond growth experiments at the laboratories of Diamond Materials. In these experiments, different growth parameters are tested in order to find the optimum combination to fulfil the target.

Following a stepwise approach, a dedicated substrate holder was first designed to grow disks of $180 \mathrm{~mm}$ 
without changing the reactor base plate. In fact, this change would have required a redesign of the cooling configuration layout in the reactor. Current reactors are able to deposit diamond on substrates only with a diameter between 120 and $150 \mathrm{~mm}$. Then, starting with growth conditions close to the ones normally used for deposition on the $150 \mathrm{~mm}$ substrate, parameters like the pressure in the reaction chamber, the gas composition and the microwave power have been varied.

For instance, it is not possible to control the shape of the plasma in the reactor. Therefore, to allow a more homogeneous diamond deposition over an enlarged area, the size of the plasma has been increased by reducing the pressure and increasing the power. On the other side, the gas composition in the chamber has been changed as it impacts the growth rate and the quality of the deposited diamond. Several deposition runs were carried out meanwhile for both large optical and thermal grade diamond disks. Most of them were grown as disks for thermal applications as, thermal grade material grows much faster than the optical one and thus the growth conditions (e.g., temperature distribution, stress formation) can be optimized in a more efficient way.

The very first two optical grade runs lasted for 400 and 350 hours resulting in rather homogeneous diamond wafers, but with thickness ranging from $\sim 0.3$ to $0.5 \mathrm{~mm}$ only. It should be noted that the thickness refers to unpolished disks, therefore it is given in a range. Unfortunately, due to the very small thickness, the two wafers did not remain in one piece after removing from the substrate. The first wafer broke in many small pieces while the second one broke in three larger pieces. From one of these pieces, a $39 \mathrm{~mm}$ disk was extracted from the very edge of the $180 \mathrm{~mm}$ disk and used for $\tan \delta$ measurements at Karlsruhe Institute of Technology (KIT) laboratories. The results are described in section 5.

Then, other deposition runs followed with an increasing average thickness. Some grown disks broke after removing from the substrate, but crack-free thin optical grade disks of $\sim 0.5 \mathrm{~mm}$ average unpolished thickness and $\sim 1.7 \mathrm{~mm}$ thick thermal grade disks were obtained. Diamond Materials has strongly carried on the parameters optimization process aiming to a more homogeneous temperature distribution able to decrease the stress formation during growth. The results were excellent as, recently, only one of ten grown thermal grade thick diamond disks broke and free-standing diamond disks of $2 \mathrm{~mm} / 180 \mathrm{~mm}$ were obtained. The disks delaminated crack-free from the substrate.

As a worldwide novelty, Fig. 4 shows a $180 \mathrm{~mm}$ thermal grade, crack-free, free-standing, diamond disk that was produced in a microwave plasma reactor with an average unpolished thickness of $\sim 2 \mathrm{~mm}$. Finally, it is also worth to mention an optical grade run of particular interest that led to a $1.7 \mathrm{~mm} / 180 \mathrm{~mm}$ disk. It was extracted from the reactor with no visible cracks and only after removing from the substrate, cracks appeared but the disk still remained as a single disk of $180 \mathrm{~mm}$. It was also sent to KIT for tan $\delta$ measurements.

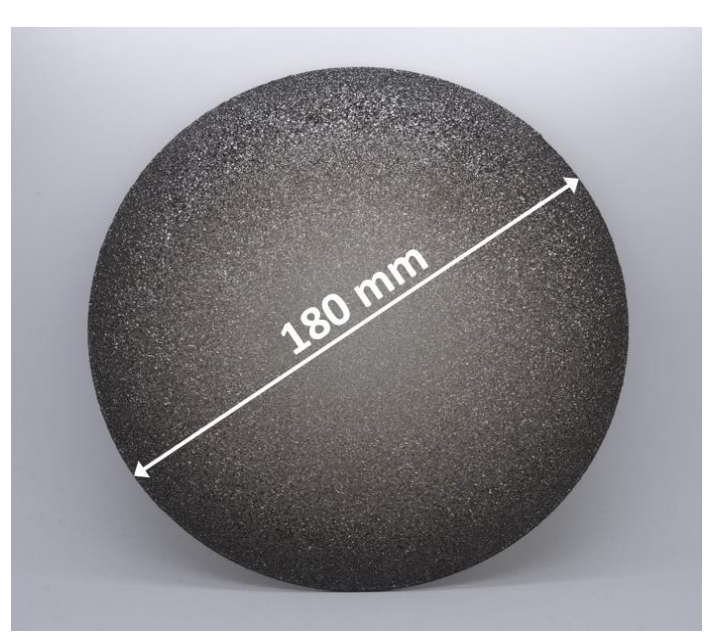

Fig. 4. The world's first free-standing thermal grade diamond disk with $180 \mathrm{~mm}$ diameter and average unpolished thickness of about $2 \mathrm{~mm}$.

\section{Loss tangent measurements of disks}

The $\tan \delta$ is measured by different open spherical and hemi-spherical Fabry-Perot resonators in dedicated test facilities at KIT [11]. These measurements, next to the diamond deposition runs, are important as they provide useful feedbacks for the optimization process of the growth parameters. In fact, the disks must have very low $\tan \delta$ values to assure low loss microwave transmission towards the plasma and consequently a low heating of the window. Measurements for bare disks may be done both at the center (where there is the maximum beam power deposition) and over the disk surface.

For the moment, using unpolished disks, even the measurement at the disk center turns out to be difficult as, in fact, without polishing there is a variation of the thickness larger than the very stringent tolerance $(\sim 5 \mu \mathrm{m})$ required by the measurement setup. For this reason, the results define only an upper limit. Using the spherical resonator shown in Fig. 5, the measurement at the center of the unpolished $39 \mathrm{~mm}$ optical disk led to a $\tan \delta<1 \mathrm{E}-04$. This result was later confirmed by the $\tan \delta$ measurement carried out at a central, crack-free, area of the $180 \mathrm{~mm}$ optical grade disk that showed cracks, but remained as a whole disk.

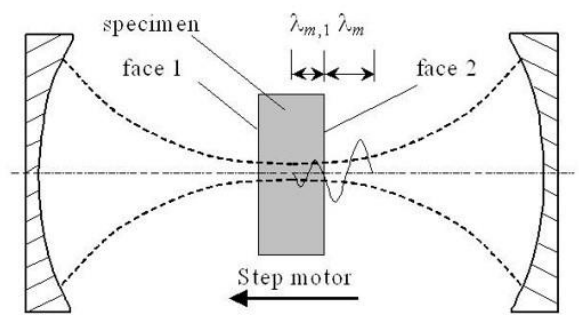

Fig. 5. Spherical Fabry-Perot resonator consisting of two spherical mirrors and the disk placed at the center. It allows high resolution measurements of $\tan \delta$ at the center of the disk. 
As a benchmark, for polished disks, the very good optical diamond disks manufactured by Diamond Materials for the ITER HCD upper launcher torus diamond windows have at the center $\tan \delta$ values lower than 2E-05. However, these disks have a thickness of $1.11 \mathrm{~mm}$ and a diameter of $75 \mathrm{~mm}$ (to be used with 50 $\mathrm{mm}$ waveguide). The obtained value of $\tan \delta<1 \mathrm{E}-04$ can be thus already considered a good result and it gives a lot of confidence that the experiments proceed in the right direction. Of course, the $\tan \delta$ measurements shall be repeated with polished optical disks.

For this reason, in the frame of 2019 WP HCD, always in collaboration with Diamond Materials, two small disks were extracted from the $180 \mathrm{~mm}$ optical unpolished cracked disk. The disks shall be polished and sent to KIT for measurements. The idea would be to make a comparison with the $\tan \delta$ of the unpolished disks, the quality of the ITER disks and finally a comparison between the quality at the center and the one at the edge of the disk.

\section{Conclusions and outlook}

The diamond Brewster-angle window is a key component required for long pulse step-frequency tuneable gyrotrons in EU DEMO reactor at $2 \mathrm{MW} \mathrm{RF}$ tube power. As for such power transmission a $63.5 \mathrm{~mm}$ WG aperture is needed, the primary target to meet is the production of large area, crack-free, optical grade diamond disks with a minimum diameter of $180 \mathrm{~mm}$ and thickness of about $2 \mathrm{~mm}$. Since $140 \mathrm{~mm}$ diameter represents the current technological limit, the path is quite complex and a very strong collaboration with the industrial diamond manufacturers is essential.

In close contact with the industrial partner Diamond Materials in Freiburg, Germany, after investigating the possibility of diamond disk joining, several optical and thermal diamond growth experiments were performed in microwave plasma reactors for $180 \mathrm{~mm}$ diameter disks. The experiments were accompanied by $\tan \delta$ measurements of the disks at KIT to help the optimization process of the growth parameters. For the very first time, $180 \mathrm{~mm}$ thermal grade, crack-free, freestanding, diamond disks were produced in a microwave plasma reactor with $\sim 2 \mathrm{~mm}$ average unpolished thickness. It thus turned out that diamond deposition on $180 \mathrm{~mm}$ substrates is feasible and, with respect to other options, it is the way to follow towards large diamond disks.

A great result was achieved, but it is also true that an intense research activity in collaboration with industry is still strongly required to meet the goal of having a feasible large area diamond disk Brewster-angle window within the DEMO conceptual design phase (2020-2027).

Next imminent steps concern the further optimization of the growth parameters for crack-free, planar, thermal grade disks and the development of proper polishing techniques. Procedures for large area disks polishing are known so far for disk diameters in the range 120$150 \mathrm{~mm}$. The acquired knowledge shall represent the base for the production of $2 \mathrm{~mm} / 180 \mathrm{~mm}$ optical grade crack-free disks.

\section{Acknowledgments}

This work has been carried out within the framework of the EUROfusion Consortium and has received funding from the Euratom research and training programme 2014-2018 and 2019-2020 under grant agreement No 633053. The views and opinions expressed herein do not necessarily reflect those of the European Commission. The authors are finally thankful to Diamond Materials for the fruitful collaboration.

\section{References}

[1] F. Albajar et al., The Development of the European $1 \mathrm{MW}$, $170 \mathrm{GHz}$ CW Gyrotron for the ITER Electron Cyclotron Heating System, Proc. 26 ${ }^{\text {th }}$ IAEA FEC, 17-22 October 2016, Kyoto, Japan, paper no. FIP/P4-29.

[2] G. Federici et al., DEMO design activity in Europe: progress and updates, Fusion Engineering and Design 136 (2018) 729-741.

[3] K.A. Avramidis et al., Overview of recent gyrotron R\&D towards DEMO within EUROfusion Work Package Heating and Current Drive, Nuclear Fusion (2019), in press.

[4] G. Aiello et al., Diamond window technology for EC heating and current drive - state of the art, Fusion Science and Technology 75 (7) (2019) 719-729.

[5] X. Yang et al., A CVD-Diamond Disk Brewster Window for a Frequency Step-Tunable 1 MW Gyrotron, Int. Journal Infrared Milli Waves, 24 (12) (2003) 2017-2024.

[6] Diamond Materials GmbH, Hans-Bunte-Str. 19, 79108 Freiburg, Germany, Website: http://www.diamondmaterials.com/EN/index.htm.

[7] G. Gantenbein et al., First Operation of a Step-Frequency Tunable 1-MW Gyrotron with a Diamond Brewster Angle Output Window, IEEE Transaction Electron Devices, 61 (6) (2014) 1806-1811.

[8] M. Thumm et al., Frequency step-tunable (114-170 GHz) megawatt gyrotrons for plasma physics applications, Fusion Engineering and Design, 53 (2001) 407-421.

[9] G. Aiello et al., Cooling Concepts for the CVD Diamond Brewster-Angle Window, Proc. 42 ${ }^{\text {nd }}$ IRMMW-THz, 27 August - 1 September 2017, Cancun, Mexico, IEEE (2017).

[10] D.C. Harris, Infrared Window and Dome Materials, SPIE, Washington, 1992.

[11] S. Schreck et al., ITER ECRH Upper Launcher: Test Plan for Qualification of the Diamond Torus Window Prototype III, Fusion Engineering and Design, 109-111 (2016) 12321236. 